Original Paper http://ajol.info/index.php/ijbcs http://indexmedicus.afro.who.int

\title{
Evaluation des techniques de production du beurre de karité au Togo
}

\author{
Kafui KPEGBA $^{1^{*}}$, Sefa Akpedjé KPOKANU ${ }^{2}$, Oudjaniyobi SIMALOU ${ }^{1}$, \\ Kosi Mawuéna NOVIDZRO ${ }^{1}$ et Kossi Honoré KOUMAGLO ${ }^{1}$ \\ ${ }^{1}$ Laboratoire des Extraits Végétaux et Arômes Naturels (LEVAN), FDS/UL, 01 BP 1515, Lomé, Togo. \\ ${ }^{2}$ Faculté des Sciences de la Santé, 01 BP 1515, Lomé, Togo. \\ *Auteur correspondant ; E-mail: donnenovi@yahoo.fr; knovidzro@univ-lome.tg; Tel: (00228) 90929862
}

\section{RESUME}

De nos jours, la filière karité a une importance socio-économique non négligeable dans le développement des grands producteurs mondiaux de beurre de karité. Le présent travail a pour objectif de faire une enquête sur les profils des unités de production, les procédés de collecte des amandes et de production de beurre, les rendements d'extraction de beurre et les caractéristiques sensorielles des beurres de karité afin de faire l'état des lieux de la filière karité au Togo. Les résultats obtenus ont montré que $31,25 \%$ des unités de production avaient recours au barattage classique, ensuite viennent les procédés de barattage Peuhl $(25,00 \%)$ et de cuisson directe $(25,00 \%)$. Les techniques industrielles (UIP1 et UIP2 : 06,25\% chacune) ainsi que le barattage sans torréfaction $(06,25 \%)$ sont les moins pratiqués. Les rendements en beurre sont de : 49,00\% (UIP1) ; 48,63\% (barattage Peuhl) ; 43,25\% (barattage classique) ; 42,00\% (UIP2), 31,33\% (barattage sans torréfaction) et $27,91 \%$ (cuisson directe). S'agissant des caractéristiques sensorielles, trois types de couleur ont été identifiés, avec une prédominance des beurres à couleur beige $(60,00 \%)$, tandis que les proportions des couleurs jaune et gris sont de $20 \%$ chacune. Cependant, la texture compacte a occupé une proportion de $93,00 \%$. Fort de ces constats, il est alors envisagé par la suite des études supplémentaires sur des paramètres physico-chimiques des échantillons afin de mieux apprécier la qualité des beurres de karité produits au Togo.

(C) 2017 International Formulae Group. All rights reserved.

Mot clés : Beurre, unités de production, procédés d'extraction, rendements en beurre, caractéristiques sensorielles.

\section{Assessment of shea butter production techniques in Togo}

\begin{abstract}
Nowadays, the shea sector has a significant socio-economic importance in the development of the world largest producers of shea butter. The objective of this study is to investigate the profiles of production units, almonds collection processes and production of butter, butter extraction yields and sensory characteristics of shea butters produced to make a critical review of the different processing methods in Togo. The results obtained showed that $31.25 \%$ of production units were using the classic churning, followed by Fulani churning processes $(25.00 \%)$ and direct cooking $(25.00 \%)$. Industrial technology (UIP1 and UIP2: 06.25\%) and churning without roasting $(06.25 \%)$ are the least practices. Butter yields found are: 49.00\% (UIP1); 48.63\%) (Fulani churning); 43.25\%
\end{abstract}


(classical churning); 42.00\% (UIP2), 31.33\% (churning without roasting) and 27, 91\% (direct cooking). Regarding the sensory characteristics, three kinds of colour have been identified, with a predominance of beige butters $(60.00 \%$, while the proportions of yellow and gray colours are $20 \%$ each one. However, the compact texture has held a proportion of $93.00 \%$. In the light of the forgoing a comprehensive study of physical and chemical parameters of the butters should be undertaken in order to better assess the quality of shea butter produced in Togo.

(C) 2017 International Formulae Group. All rights reserved.

Keywords: Butter production units, methods of extraction, butter yields, sensory characteristics.

\section{INTRODUCTION}

Le beurre de karité est une graisse extraite des amandes du fruit de l'arbre à karité (Vitellaria paradoxa, Gaertn C. F.), un arbre de la famille des Sapotacées, pouvant atteindre une quinzaine de mètres de haut et qui pousse exclusivement dans les savanes arborées d'Afrique (Yé et al., 2007). Les fruits de karité sont des baies charnues renfermant une noix dont la teneur en matière grasse se situe entre 43 et 55\% (Bourlet, 1950). L'aire écologique de Vitellaria paradoxa correspond au climat soudano-sahélien avec une pluviométrie allant jusqu'à $1000 \mathrm{~mm}$ (Kassamba, 1997). On dénombre 16 pays producteurs, formant une ceinture de 5000 $\mathrm{km}$ et s'étendant de la région Ouest jusqu'à l'Est du Sud Soudan (Maranz and Wiesman, 2003 ; Masters, 2004). Cette ceinture appelée « ceinture de karité » par les commerçants (Ferris, 2001) intègre les pays suivants : le Bénin, le Burkina Faso, le Cameroun, la République Centrafricaine, la Côte d'Ivoire, la Gambie, le Ghana, la Guinée, le Mali, le Niger, le Nigeria, l'Ouganda, le Sénégal, le Sud Soudan, le Tchad et le Togo (Chalfin, 2004 ; Goreja, 2004).

Vitellaria comporte une seule espèce, Vitellaria paradoxa, avec deux sous-espèces : Vitellaria paradoxa paradoxa et Vitellaria paradoxa nilotica (Hall et al., 1996). La sousespèce paradoxa se trouve dans la zone ouest de la région du karité qui s'étend sur $4700 \mathrm{~km}$ et couvre 14 pays dont le Togo, tandis que la sous-espèce nilotica occupe la zone orientale. Le beurre de la sous-espèce occidentale, paradoxa, est riche en acide stéarique et donne un beurre ferme, alors que la graisse issue de la sous-espèce orientale nilotica est surtout riche en acide oléique donnant une huile liquide (Bonkoungou, 2002).

En 2005, la production mondiale en noix de karité était estimée à près de 700000 tonnes, les principaux producteurs étant le Nigéria (61\%), le Mali (12\%) et le Burkina Faso (10\%). Les exportations sont surtout constituées d'amandes séchées qui sont ensuite transformées en Europe et aux USA de manière industrielle par extraction chimique ou par pressage mécanique (Yé et al., 2007). Et selon les estimations de 2004, le Togo a récolté 15000 tonnes d'amandes et produit la même quantité de beurre de karité (Lovett, 2004). Le beurre de karité est très apprécié dans les industries alimentaires (huilerie, chocolaterie), cosmétiques (hydratation de la peau, protection contre les UV, etc.) et pharmaceutiques (cicatrisation des blessures, etc.) (Soro et al., 2011 ; Gnangle et al., 2012, Kouyate et al.,2015), pour ses propriétés intrinsèques liées à sa composition en glycérides et sa teneur élevée en insaponifiables (Kapseu et al., 2001), ce qui lui offre de nombreux débouchés sur les marchés internationaux (Yé et al., 2007). En effet, il a été démontré que le beurre de karité possède de réelles vertus pour la peau; il hydrate, adoucit, protège et embellit grâce à sa composition chimique exceptionnellement riche en insaponifiables, en vitamines (A, D, E, F) et en latex. C'est ainsi qu'il est utilisé dans les pays africains par les mères qui enduisent leurs bébés de ce beurre pour prévenir ou guérir les endroits irrités. D'autre part, le massage des muscles avec ce beurre avant et après l'effort physique leur apporte la souplesse et permet une meilleure 
récupération, d'où son utilisation par la plupart des sportifs africains.

En plus des effets biologiques, le beurre de karité présente aussi des avantages économiques pour de nombreux pays en développement. Dès lors, la récolte et la transformation des produits dits forestiers non-ligneux, comme le beurre de karité, deviennent une réelle opportunité pour les populations de ces pays, notamment les femmes (Aboubakar et al., 2009). Les parcs à karité doivent donc leur existence et leur structure à l'homme (Diarassouba et al., 2008). Mais il est fréquent de constater l'élimination de l'arbre, notamment lorsque des terres sont défrichées au profit de la culture du coton et de l'igname, de même que pour la production de charbon (Tchabi et Adechi, 2014).

Parmi les secteurs «à fort potentiel» au Togo, la filière karité se place en bonne position. La valorisation de cette filière devient alors un impératif de développement. En outre, si cette filière a fait l'objet de nombreuses études dans des pays voisins comme le Burkina Faso, le Ghana et dans d'autres comme le Mali, le Nigeria et la République Centrafricaine (FAO, 2001; Mbétid-Bessane, 2005), au Togo rien n'a été fait dans ce sens jusqu'à présent. C'est pour ce besoin que la meilleure gestion du karité en vue de rendre durable les bienfaits et bénéfices inestimables, devra se faire en collaboration étroite avec les acteurs concernés. La valorisation du karité et l'organisation de la filière nécessitent donc avant toute chose de faire un état des lieux concernant la filière karité au Togo.

$\mathrm{Ce}$ travail avait donc pour objectif d'évaluer les techniques de production du beurre de karité au Togo en vue de faire un état des lieux dans la filière.

\section{MATERIEL ET METHODES}

Visite des unités de production

Les travaux ont été effectués sur des échantillons de beurre de karité collectés dans 13 localités dont 12 situées dans les trois principales régions de production de beurre de karité au Togo (Centrale, Kara et Savanes) et une (Katanga), située dans la région Maritime. Il a été exploré, 10 unités de production artisanales, une société spécialisée dans la production (AGBANGA KARITE) et deux unités industrielles de production (UIP1 et UIP2) (Tableau 1).

\section{Matériel végétal}

Les travaux ont été effectués avec 15 échantillons de beurre de karité collectés dans les 13 localités situées dans les quatre régions du Togo concernées par notre étude (Tableau 1).

\section{Méthodes}

L'étude a été réalisée en deux phases : la première a consisté à faire une enquête dans les différentes unités de production visitées, couplée simultanément avec la collecte des échantillons de beurre de karité à étudier et la seconde a été consacrée à une analyse des caractéristiques sensorielles des échantillons collectés.

\section{Enquête dans les unités de production}

Cette enquête a été conduite du $1^{\mathrm{er}}$ au 31 Décembre 2015. Le choix des localités cibles a été effectué selon la méthode d'Aboubakar et al. (2009) qui consiste à privilégier comme zones d'études les localités où il existe un grand peuplement naturel de karité et une tradition de transformation des amandes en beurre.

Pour le recueil des données, des questionnaires semi-directifs ont été utilisés. Les principaux points abordés dans ces questionnaires sont: identification des producteurs ; procédés d'obtention des fruits, leurs conditionnement, stockage et conservation; débouchés des fruits collectés, différents procédés de transformation des amandes en beurre; quantités moyennes saisonnières d'amandes transformées en beurre; rendements d'extraction en beurre ; conditionnement, stockage et conservation du beurre et débouchés du beurre produit.

\section{Collecte des échantillons}

Les entretiens se sont déroulés sur les lieux de production suivis de la collecte des 
échantillons de beurre de karité. Les échantillons ont été recueillis dans des bocaux en matière plastique dans des conditions rigoureuses d'hygiène et conservés au laboratoire à une température d'environ - 23 ${ }^{\circ} \mathrm{C}$. Lorsque cela a été possible, nous avions suivi directement les différentes étapes de production du beurre. Dans les meilleurs cas, les échantillons de beurre prélevés étaient ceux fraîchement produits le jour de notre enquête. Dans le cas échéant, les échantillons collectés étaient ceux issus de la production la plus récente. Chaque échantillon a été identifié par un numéro alpha-numérique, constitué des deux premières lettres de la préfecture d'origine où il est prélevé, et suivi par le numéro d'ordre de collecte. Les différents sites de production que nous avons prospectés sont enregistrés dans Tableau 1.

\section{Détermination des caractéristiques sensorielles}

L'évaluation des caractéristiques sensorielles a été effectuée immédiatement après la collecte des échantillons. Cela a consisté à une appréciation de la couleur, de la texture ainsi que du parfum de chaque échantillon collecté. Ces caractéristiques sont définies suivant le test sensoriel décrit par Konan et al. (2003). Cependant, la seule modification apportée a été le remplacement du caractère " goût» par le caractère «parfum», étant donné que le beurre n'est pas en général un produit destiné à la consommation. Toutefois, nous n'avons pas eu recours à des testeurs qualifiés. En effet :

$\checkmark$ la couleur du beurre a été définie après avoir déposé une noisette de beurre de karité sur une feuille de papier rame de couleur blanche ;

$\checkmark$ la texture quant à elle a été déterminée en écrasant, entre le pouce et l'index, l'équivalent d'un gramme de beurre de karité. Le beurre est dit fondant, lorsqu'il fond à la première friction, et compact, dans le cas contraire ;

$\checkmark \quad$ le parfum a été défini en humant du beurre étalé entre les doigts. Il est dit fort, lorsque le beurre exhale le parfum caractéristique du karité sans besoin de friction. Il est considéré comme moyen, s'il ne se perçoit qu'après friction entre les doigts, et faible, lorsqu'il est à peine perceptible, même après friction. Le beurre est dit ranci, dans le cas où son parfum est ocre et piquant aux narines.

Tableau 1 : Points de collecte des données de terrain et identification des échantillons collectés.

\begin{tabular}{|c|c|c|c|}
\hline REGIONS & PREFECTURES & LOCALITES & $\mathrm{N}^{\circ}$ DES ECHANTILLONS \\
\hline \multirow{5}{*}{ KARA } & Assoli & $\begin{array}{l}\text { Agbebou * } \\
\text { Agbang * }\end{array}$ & $\begin{array}{l}\text { ASO1 } \\
\text { KZ03 }\end{array}$ \\
\hline & Kozah & Kougouyo * & KZ04 \\
\hline & & Atétou * & KE05 \\
\hline & Kéran & Anima * & KE06 \\
\hline & Bassar & Bassar * & BA14 \\
\hline \multirow{4}{*}{ SAVANES } & Tandjouaré & Tandjouaré * & TA08, TA09 \\
\hline & Cinkassé & Cinkassé * & CI10, CI11 \\
\hline & $O$ & Mango * & OT12 \\
\hline & & Tamonga (UIP1) $* * *$ & OT17 \\
\hline \multirow[b]{2}{*}{ CENTRALE } & Blitta & Blitta * & $B L 15$ \\
\hline & Tchaoudjo & - Filandi-Somou & $T C 16$ \\
\hline MARITIME & Golfe & - Katanga (UIP2) $* * *$ & GO18 \\
\hline
\end{tabular}

*: Unité de production artisanale $\quad * *$ : Société spécialisée $\quad * * *$ UIP1/ UIP2 : Unité Industrielle de Production 1 et 2 


\section{RESULTATS}

Profils des unités de production

D'après nos enquêtes, les profils des différentes unités de production de beurre de karité au Togo sont fournis dans le Tableau 2.

\section{Procédés d'obtention des amandes}

Notre enquête de terrain nous a permis de distinguer quatre procédés d'obtention d'amandes de karité au Togo (Figure 1).

Dans le procédé A, les fruits, une fois ramassés, sont dépulpés à la main. Les noix obtenues, après dépulpage, subissent une cuisson, puis un séchage au soleil après lequel elles sont décortiquées pour libérer les amandes. Ensuite, les amandes libérées sont séchées au soleil avant d'être stockées dans des sacs ou des greniers.

Dans le procédé $\mathrm{B}$, le dépulpage des fruits est précédé d'une fermentation en fosse des fruits. Les noix obtenues ne sont pas cuites mais séchées directement au soleil puis décortiquées. Les amandes obtenues après décorticage subissent un séchage au soleil comme dans le procédé A. Elles sont ensuite stockées dans des sacs ou en grenier.

Les procédés $\mathrm{C}$ et $\mathrm{D}$ impliquent un dépulpage manuel des fruits. Dans le procédé $\mathrm{C}$, les noix subissent une cuisson puis un séchage au four alors que dans le procédé $\mathrm{D}$, elles sont directement séchées au four. Dans ces deux procédés, les noix ne sont pas décortiquées, mais stockées telles quelles dans des sacs ou des greniers.

\section{La répartition des procédés d'obtention des} amandes

La répartition de la collecte des amandes est illustrée sur la Figure 2.

Procédés de production de beurre de karité

Les procédés avec lesquels la production de beurre de karité se réalise au Togo sont enregistrés sur la Figure 3. En ce qui concerne les procédés industriels, ils sont représentés par ceux des huileries UIP1 et UIP2 (Figure $4: A$ et $B$ ).

\section{La répartition des techniques de} production de beurre de karité

Les résultats relatés dans le Tableau 3 concernent la répartition des techniques de production de beurre de karité au Togo.

\section{Rendements d'extraction de beurre}

D'après les données recueillies, à l'issue de nos enquêtes, les rendements d'extraction de beurre de karité dans les différentes unités de production au Togo sont consignés dans le Tableau 4.

\section{Caractéristiques sensorielles}

D'après notre enquête, les Figures 5-A, 5-B et 5-C affichent les caractéristiques sensorielles de l'ensemble des différentes sortes de beurre produit au Togo.

Tableau 2 : Répartition des unités de production.

\begin{tabular}{|c|c|c|c|}
\hline Unité de production & Productrices d'amandes & Non productrices d'amandes & Total \\
\hline \multirow{2}{*}{ huileries $\left\{\begin{array}{l}\text { Industrielles } \\
\text { traditionnelles }\end{array}\right.$} & 0 & 2 & 2 \\
\hline & 0 & 1 & 1 \\
\hline Coopératives & 4 & 1 & 5 \\
\hline Particuliers & 2 & 3 & 5 \\
\hline Total & 6 & 7 & 13 \\
\hline
\end{tabular}



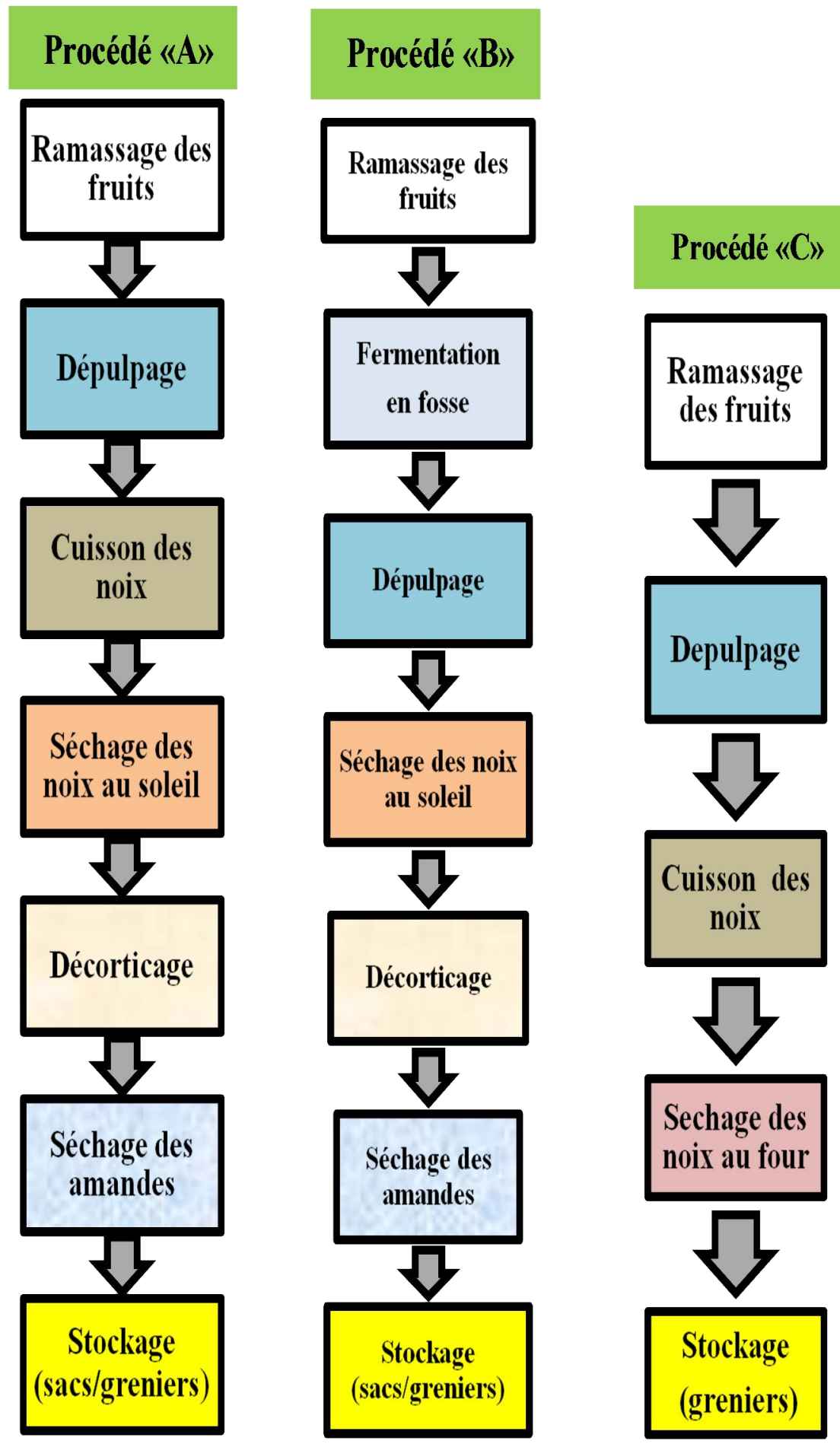

\section{Procédé «D»}

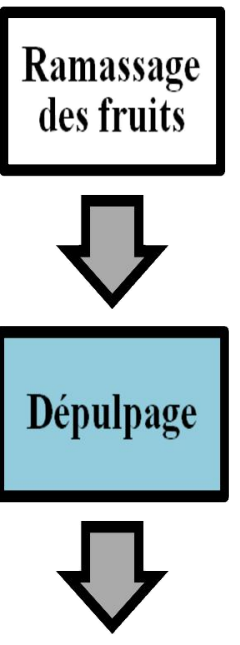

Séchage des noix au four

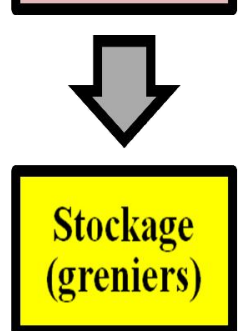

Figure 1 : Procédés d'obtention des amandes de karité au Togo. 


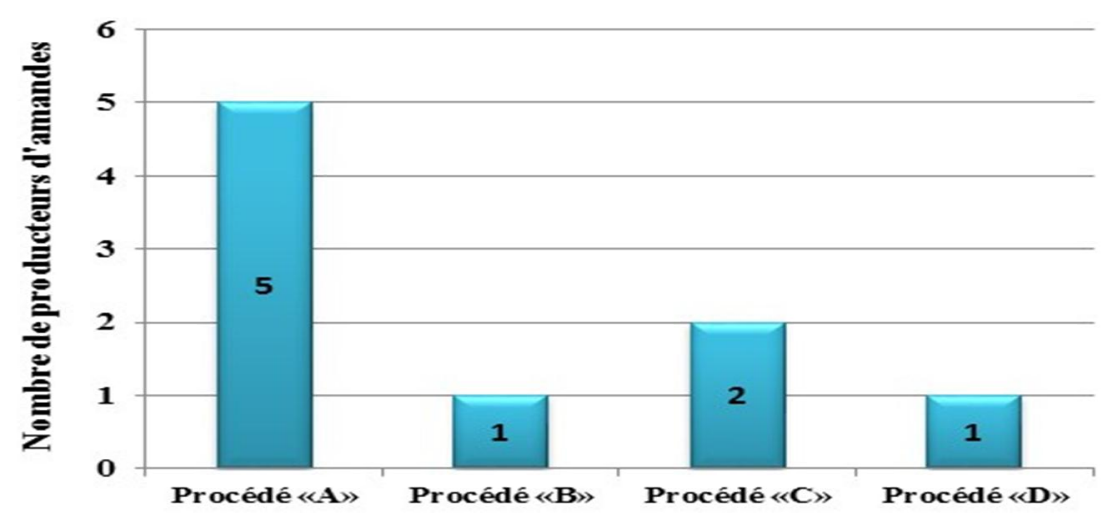

Figure 2 : Répartition des procédés de production d'amandes.
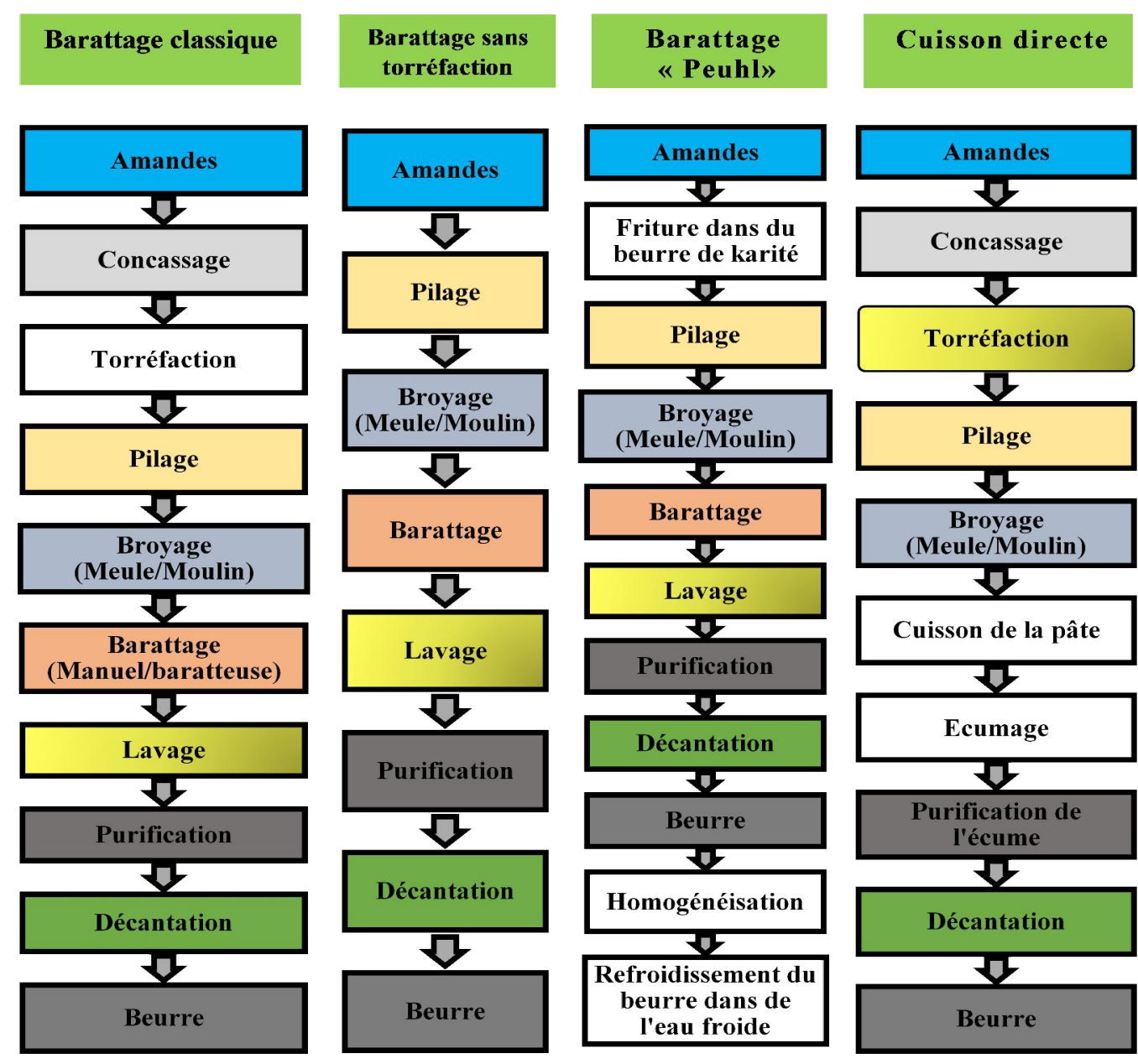

: Facultatif

Figure 3 : Procédés traditionnels de production de beurre de karité au Togo. 


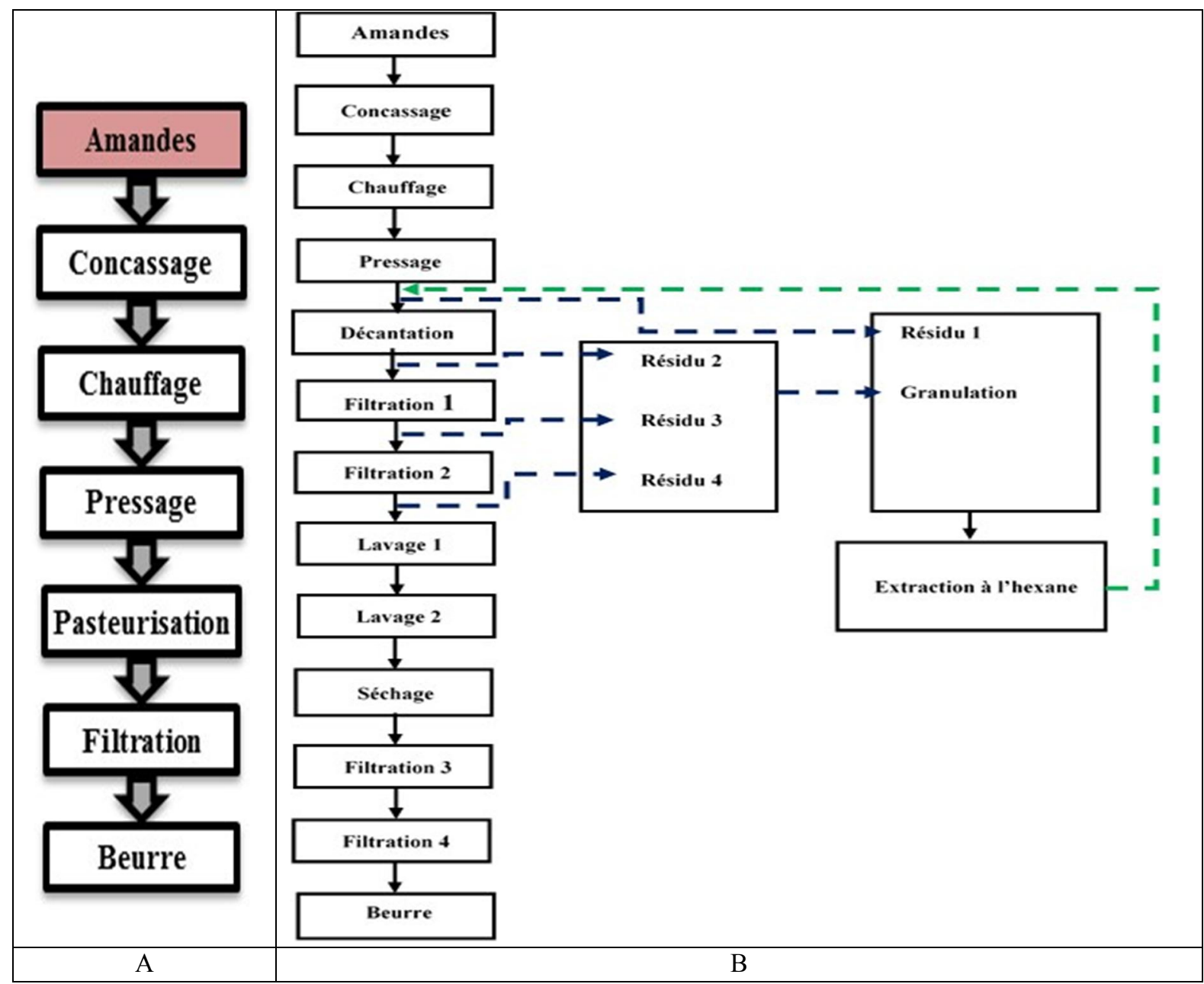

Figure 4 : Procédés industriels d'extraction de beurre de karité au Togo dans les huileries UIP1(A) et UIP2 (B).

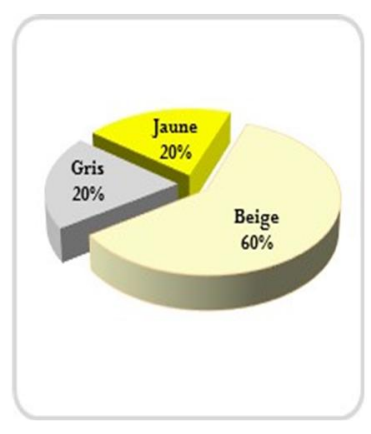

A

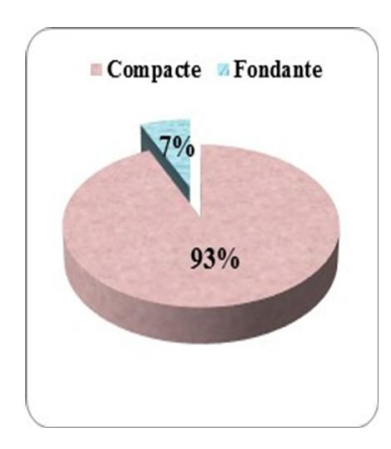

B

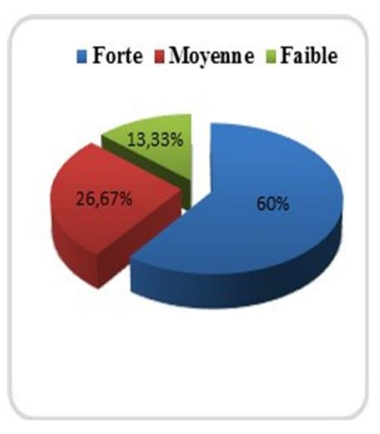

C

Figure 5: Répartitions des échantillons en fonction : des couleurs (A); des textures (B) et des parfums (C). 


\section{DISCUSSION}

\section{Profils des unités de production}

Au total, 13 unités de production ayant des profils regroupés dans le Tableau 2, ont été prospectées. Ces différents profils reflètent donc la faible couverture industrielle de production de beurre de karité au Togo. Les données du Tableau 2 nous montrent que 12 unités de production ont été retrouvées dans les trois principales régions de production de beurre de karité au Togo parmi lesquelles 10 sont de type traditionnel, une est de type traditionnel (huilerie de la société AGBANGA KARITE) et une autre de type industriel (huilerie UIP1). La $13^{\mathrm{e}}$ unité de production, située dans la région Maritime, est également une huilerie de type industriel (UIP2) (Tableau 1). Il est à noter que les unités de production de type traditionnel sont tenues soit par des particuliers, ou soit par des coopératives.

On a pu remarquer que 6 unités, sur les 13 au total, sont également productrices d'amandes destinées à l'extraction du beurre de karité.

\section{Procédés d'obtention des amandes}

Le procédé A (Figure 1) présente des similitudes avec la technique d'obtention des amandes décrite par Ahouannou et al. (2003) au Bénin dans les procédés Peuhl et Lokpa. Cependant, dans les procédés $\mathrm{A}$ et $\mathrm{C}$, les noix subissent une cuisson, alors que le procédé $\mathrm{D}$ implique leur séchage au four. Toutefois, la pratique de la cuisson ou du séchage au four peut porter des graves préjudices à la qualité $\mathrm{du}$ beurre produit, comme par exemple la formation des composés aromatiques. Par contre, selon Kaboré et Gadiaga (1992), Gadiaga (1996), Kassamba (1997), la cuisson, tout comme le séchage des noix au four, permet de bloquer la germination des graines. En effet, Karo et al. (1988) ont affirmé que la germination des graines réduit le rendement d'extraction du beurre et développe une amertume de celui-ci, occasionnant ainsi une dégradation de la qualité du beurre.

Masters (2002) et Lovett (2004) de leur part, ont aussi constaté, d'après leurs études, que le séchage au four peut provoquer la formation de grandes quantités d'hydrocarbures polycycliques aromatiques connus pour être cancérigènes.

Dans le procédé B (Figure 1), les fruits sont dépulpés après un processus de fermentation en fosse. D'après Masters (2002), les fortes températures engendrées par ce processus participent à une dénaturation des enzymes de croissance en empêchant la germination des graines, minimisant ainsi l'oxydation enzymatique. Malheureusement, ce processus peut donner à l'amande un parfum déplaisant. Par conséquent, ce procédé n'est plus encouragé aujourd'hui.

Toutefois, le procédé B n'implique aucun processus thermique. Les noix, toutes comme les amandes, sont simplement séchées au soleil. Mais d'après les investigations menées par Kapseu (2009) et Lovett (2004), le séchage direct au soleil est une technique lente, soumise aux conditions climatiques entraînant des risques de pollution et d'oxydation élevés. Il est néanmoins favorable à l'action des lipases, entrainant ainsi l'augmentation des acides gras libres dans le beurre.

\section{La répartition des procédés d'obtention des amandes}

Le procédé par barattage classique pratiqué au Togo (Figure 3) présente des similitudes avec la technique de production de beurre de karité dans le procédé Lokpa au Bénin, décrite par Ahouannou et al. (2013). $\mathrm{Au}$ cours de ce procédé, les amandes sont torréfiées avant l'extraction proprement dite. Selon Karleskind (1992), le traitement thermique des amandes favorise la rupture des lipocytes, contribuant ainsi à une amélioration du rendement d'extraction du beurre.

Le barattage sans torréfaction et la cuisson directe (Figure 3) sont respectivement similaires aux techniques de production des procédés « par voie humide " et " par voie sèche » décrites par Aboubakar et al. (2009) au Cameroun. La cuisson directe présente aussi des similitudes avec le procédé de 
Garoua au Cameroun décrit par Nkouam (2007).

Le procédé « Peuhl » décrit dans notre étude (Figure 3 ) présente de nombreux points communs avec la technique de production de beurre de karité au Bénin décrite par Ahouannou et al. (2013). La différence entre ces deux procédés réside dans le fait que, dans notre étude, le beurre, une fois extrait et purifié, est homogénéisé, puis mis en contact direct avec de l'eau froide afin d'accélérer son durcissement, alors que dans le procédé Peuhl du Bénin, cette pratique n'intervient pas.

Dans l'huilerie UIP1, c'est le procédé d'extraction par pressage à froid qui est employé, alors que dans UIP2, c'est le pressage à chaud complété par une extraction à l'hexane qui est mis en pratique.

\section{Procédés de production de beurre de karité}

Les Figures 3 et 4 montrent que, pour produire le beurre de karité au Togo, les producteurs font recours à la fois aux procédés traditionnels et industriels. S'agissant des procédés traditionnels, quatre types se pratiquent : le barattage classique et celui sans torréfaction, le barattage « Peuhl » et celui de cuisson directe. Au niveau des procédés traditionnels, quatre types existent : le procédé de barattage classique et ceux de barattage sans torréfaction, de barattage « Peuhl» et de cuisson directe.

$\checkmark$ Le procédé de barattage classique implique un concassage, une torréfaction, un pilage puis un broyage (à la meule traditionnelle ou au moulin) des amandes de karité. La pâte obtenue est barattée. Le surnageant obtenu est parfois lavé avant d'être purifié, puis décanté.

$\checkmark$ Quant au procédé de barattage sans torréfaction, il est semblable au barattage classique sauf que le concassage et la torréfaction des amandes n'y sont pas effectués.

$\checkmark \quad$ Dans le procédé de barattage «Peuhl », les amandes sont frites dans du beurre de karité avant d'être pilées et broyées. La pâte obtenue après broyage subit un barattage et le surnageant obtenu subit le même processus que dans le barattage classique. Cependant, le beurre obtenu est homogénéisé à l'aide d'une spatule en bois puis refroidi par contact direct avec de l'eau froide afin d'accélérer son durcissement.

$\checkmark$ Dans le procédé par cuisson directe, les amandes sont concassées et parfois torréfiées. Elles sont ensuite pilées puis broyées. La pâte obtenue est ensuite cuite et écumée. L'écume est ensuite purifiée et décantée pour aboutir finalement au beurre de karité.

$\checkmark$ Dans l'huilerie UIP1, c'est le procédé d'extraction par pressage à froid qui est employé, alors qu'au sein de l'huilerie UIP2, les amandes sont concassées, chauffées aux températures variant entre $110{ }^{\circ} \mathrm{C}$ et $120{ }^{\circ} \mathrm{C}$. Elles sont ensuite pressées et l'huile obtenue est dirigée vers les bacs à décantation, alors que les tourteaux d'extraction sont épuisés en huile par extraction à l'hexane. L'huile extraite à l'hexane est aussi dirigée vers les bacs à décantation où elle se mélange à l'huile de pressage. Ce mélange après décantation subit quatre filtrations, lavages et séchages avant d'être stocké.

\section{La répartition des techniques de production de beurre de karité}

La répartition des techniques de production de beurre de karité dans les zones cibles visitées (Tableau 3) a montré que le barattage classique était employé par cinq unités de production artisanale, soit 31,25\% des unités enquêtées. De ce fait, le barattage classique apparaît comme le procédé le plus utilisé dans la production de beurre de karité au Togo. En seconde position, viennent les procédés de barattage Peuhl $(25,00 \%)$ et de cuisson directe $(25,00 \%)$. Tandis que les techniques industrielles des huileries UIP1 et UIP2 ainsi que le procédé de barattage sans torréfaction sont les moins pratiqués, avec un pourcentage de $06,250 \%$ pour chacun.

\section{Rendements d'extraction de beurre}

Dans l'ensemble, les données du Tableau 4 montrent que les procédés à meilleurs rendements ont été ceux de : UIP2 $(42,00 \%)$, barattage classique $(43,25 \%)$, barattage Peuhl $(48,63 \%)$ et UIP1 $(49,00 \%)$, 
tandis que les faibles rendements sont remarqués au niveau de la cuisson directe $(27$, $91 \%)$ et du procédé de barattage sans torréfaction $(31,33 \%)$.

Le faible rendement fourni par la technique de cuisson directe $(27,91 \%)$ pourrait s'expliquer par le fait que cette technique consomme beaucoup de bois de chauffe qui est actuellement une ressource précieuse en milieu rural, surtout dans les régions sahéliennes. De ce fait, il semblerait que par souci d'économie, les producteurs ne mènent pas le processus de chauffage jusqu'à terme. Du coup, l'épuisement total en huile du fond résiduel ne serait pas donc bien assuré. Quant au barattage sans torréfaction, son faible rendement $(31,33 \%)$ pourrait être dû à l'absence de prétraitement thermique des amandes lors du processus d'extraction. En effet, selon certaines études (Wolf, 1992 ; Lanoiselle et Bouvier, 1994), le traitement thermique des amandes permet d'améliorer le rendement d'extraction. En ce qui concerne le barattage Peuhl, le refroidissement du beurre par contact direct avec de l'eau froide, afin d'accélérer son durcissement, serait probablement à l'origine de ce rendement si élevé (48,63\%), obtenu à cause de l'absorption d'eau par le beurre. Ce qui peut probablement porter des atteintes à la qualité de ce type de beurre, car une teneur en eau très élevée peut provoquer facilement le rancissement du beurre.

\section{Caractéristiques sensorielles}

Trois types de couleur ont été identifiés pour l'ensemble de nos échantillons, avec une prédominance des beurres à couleur beige qui ont occupé une proportion de $60 \%$, tandis que les proportions des couleurs jaune et gris sont de $20 \%$ chacune (Figure 5-A). Cependant pour conserver ces couleurs initiales, Dahouenon-Ahoussi et al. (2012) ont envisagé que des précautions de pré-raffinage et de conditionnement doivent être prises afin de limiter une dénaturation ultérieure qui conduirait à une décoloration des corps gras.

En outre, la détermination des textures (Figure 5-B) a révélé une prédominance de la texture compacte avec une proportion de $93 \%$, tandis que la Figure 5-C montre que les échantillons de beurre étudiés à fort parfum ont représenté $60 \%$ par rapport à l'ensemble des échantillons analysés.

La diversité des caractéristiques sensorielles des différents échantillons de beurres de karité produits au Togo pourrait s'expliquer par la multiplicité des méthodes d'extraction employées au sein des différentes unités de production. Dans leurs études respectives, Hall et al. (1996), Kapseu et al. (2005), ainsi que Womeni et al. (2006), imputaient ces variations à celles des températures et durées de torréfaction des amandes.

En effet, d'après Bayala (2002), la torréfaction a tendance à intensifier le parfum naturel des noix, avec pour résultat, un arôme peu approprié aux applications à forte valeur (cosmétiques et pharmaceutiques). A propos de la texture, Maranz et al. (2004) attribuaient la texture compacte de la plupart des beurres de karité ouest-africains à sa forte teneur en acide gras saturés.

Tableau 3 : Répartition des techniques de production de beurre de karité.

\begin{tabular}{llllc}
\hline Techniques & & n & Pourcentages (\%) \\
\hline Traditionnelles & & Classique & 5 & 31,25 \\
& Barattage & Sans torréfaction & 1 & 06,25 \\
& & Peuhl & 4 & 25,00 \\
\cline { 2 - 5 } & Cuisson directe & & 4 & 25,00 \\
\hline \multirow{2}{*}{ Industrielles } & UIP1 & & 1 & 06,25 \\
& UIP2 & 1 & 06,25 \\
\hline
\end{tabular}

$\mathrm{n}=$ nombre d'unités de production 
Tableau 4 : Rendements d'extraction de beurre.

\begin{tabular}{lllc}
\hline Techniques & & & Rendement (\%) \\
\hline Traditionnelles & & Classique & 43,25 \\
& \multirow{2}{*}{ Barattage } & Sans torréfaction & 31,33 \\
& & Peuhl & 48,63 \\
\cline { 2 - 4 } & Cuisson directe & & 27,91 \\
\hline \multirow{2}{*}{ Industrielles } & UIP1 & 42,00 \\
& UIP2 & & 49,00 \\
\hline
\end{tabular}

\section{Conclusion}

Cette étude avait pour objectif de faire un état des lieux des techniques de production et de de déterminer des caractéristiques sensorielles du beurre de karité produit au Togo. A l'issue d'une enquête ethnobotanique effectuée, au total 13 unités de production ont été explorées, pratiquant dans la production de beurre de karité, deux grands types de procédés (traditionnels et procédés industriels). Cependant, l'analyse des profils des différentes unités de production a montré une prédominance des procédés traditionnels. Aussi, quatre différentes méthodes de collecte des amandes étaient utilisées. D'après les données recueillies, une proportion de $31,25 \%$ des unités de production avaient recours au barattage classique, ensuite suivaient les procédés de barattage Peuhl $(25,00 \%)$ et de cuisson directe $(25,00 \%)$. Les techniques industrielles des huileries UIP1 et de UIP2 $(06,25 \%)$ ainsi que le procédé de barattage sans torréfaction $(06,25 \%)$ sont les moins appliquées. De ces différents procédés, les meilleurs rendements d'extraction de beurre proviennent du procédé industriel de UIP2 (42,00\%), du barattage classique $(43,25 \%)$, de barattage Peuhl $(48,63 \%)$ et surtout du procédé industriel de UIP1 $(49,00 \%)$. Par contre, les faibles rendements ont été obtenus $\mathrm{du}$ barattage sans torréfaction $(31,33 \%)$ et surtout de la cuisson directe $(27,91 \%)$. S'agissant des caractéristiques sensorielles, trois types de couleur ont été identifiés, avec une prédominance des beurres à couleur beige $(60,00 \%)$; tandis que la texture compacte a occupé une proportion de $93,00 \%$. Cependant, les beurres à fort parfum ont représenté $60,00 \%$ de l'ensemble des échantillons analysés.

Ainsi, compte tenu des différentes techniques de production et de la diversité des caractéristiques sensorielles du beurre de karité produit au Togo, il est à envisager, par la suite, une étude complémentaire pour déterminer les paramètres physico-chimiques de ce produit afin de mieux apprécier sa qualité.

\section{CONFLITS D'INTERETS}

Tous les auteurs déclarent qu'il n'existe aucun conflit d'intérêts concernant la publication de cet article.

\section{CONTRIBUTIONS DES AUTEURS}

KK a participé à la conception du sujet, à la recherche des fonds d'appui et à la supervision du travail. SAK a fait la visite des sites et la collecte des échantillons de beurre, a réalisé les enquêtes dans les unités de production, puis a fait les analyses et a interprété les résultats. OS a participé à l'élaboration des fiches d'enquêtes et à la correction du manuscrit. KMN a participé à l'interprétation des résultats et à la rédaction $\mathrm{du}$ manuscrit, puis à sa soumission pour publication et aux corrections souhaitées par les reviwers. KHK a participé à la mise à 
disposition de son laboratoire de recherche et à la validation des résultats.

\section{REMERCIEMENTS}

Les auteurs remercient très sincèrement le Service de Coopération d'Action Culturelle (SCAC) de l'Ambassade de France au Togo pour son soutien financier pour la mise en œuvre de ce projet.

\section{REFERENCES}

Aboubakar DAK, Adjia HZ, Kameni A, Tchiégang C. 2009. Procédés traditionnels de production et circuit de commercialisation du beurre de karité au Nord-Cameroun. Tropicultura, 27: 3-7.

Ahouannou C, Tchobo FP, Toukourou CA, Kougbadi F, Soumanou MM. 2013. Influence des opérations thermiques impliquées dans les procédés traditionnels d'extraction du beurre de karité au Bénin. Int. J. Biol. Chem. Sci., 7(5) : 2151-2164.

Bayala J. 2002. Rôle des institutions de recherche dans le secteur du karité (Vitellaria paradoxa Gaertn C.F.) : acquis scientifiques et perspectives, Atelier FAO (Food and Agriculture Organization) /CFC (Common Fund for Commodities) sur le traitement, la valorisation et le commerce du karité en Afrique, Sénégal : Centre de suivi écologique Dakar, (du 4 au 6 mars 2002), p.128-148.

Bonkoungou EG. 2002. Actes de l'atelier international organisé par l'Organisation des Nations Unies pour l'Alimentation et l'Agriculture, le Fonds Commun pour les Produits de Base et le Centre de Suivi Ecologique, portant sur: Le traitement, la valorisation et le commerce du karité en Afrique: L'arbre à karité (Vitellaria paradoxa) et les parcs à karité en Afrique, Dakar, Sénégal (du 4 au 6 mars 2002).
Bourlet G. 1950. Le problème du karité Oléagineux, 5(6): 364-367.

Chalfin B. 2004. Shea Butter Republic. Rout Ledge: New York.

Dahouenon-Ahoussi E, Djenontin TS, Codjia DRM, Tchobo FP, Alitonou AG, Dangou J, Avlessi F, Sohounhloue DCK. 2012. Morphologie des fruits et quelques caractéristiques physique et chimique de l'huile et des tourteaux de Irvingia gabonensis (Irvingiaceae). Int. J. Biol. Chem. Sci., 6(5): 2263-2273. http://dx.doi.org/10.4314/ijbcs.v6i5.32

Diarassouba N, Koffi KE, N'Guessan KA, Van Damme P, Sangare A. 2008. Connaissances locales et leur utilisation dans la gestion des parcs à karité en Côte d'Ivoire. Afrika Focus, 21: 77 - 96.

FAO. 2001. Les produits forestiers nonligneux en Afrique : un aperçu régional et national. Document de travail FOPW/01/1 (S. Walter, Ed.), Département des Forêts, FAO : 303p.

Ferris RSB, Collinsom C, Wanda K, Jagwe J, Wright P. 2001. Evaluating the Market Opportunities for Shea Nut and Shea Nut Processed Products in Uganda. Submitted to United States Agency for International Development (USAID).

Gadiaga A. 1996. Le traitement du karité du fruit au beurre : la conservation et la transformation, méthodes utilisées au Burkina Faso, Atelier international d'échanges " Oléagineux et petites technologies », ENSAI Ngaoundéré, APICA, Douala, p.92-117.

Gnangle PC, Egah J, Baco MN, Gbemavo CDSJ, Kakaï RG, Sokpon N. 2012. Perceptions locales du changement climatique et mesures d'adaptation dans la gestion des parcs à karité au NordBénin. Int. J. Biol. Chem. Sci., 6(1): 136149. DOI: http://dx.doi.org/10.4314/ ijbcs.v6i1.13 
Goreja WG. 2004. Shea Butter: The Nourishing Properties of Africa's BestKept Natural Beauty. Amazing Herbs Press: New York; 53.

Hall JB, Aebixcher DP, Tomlinson HF, OseiAmaning E, Hindle JR. 1996. Vitellaria paradoxa (Number 8). School of Agricultural and Forest Sciences Publication, University of Wales: Bangor; 1-105.

Kaboré TH, Gadiaga G. 1992. Le karité au Burkina Faso, transformer ou pas, le difficile choix des productrices. TPA., 5: 6-9.

Kapseu C, Jiokap NY, Parmentier M, Dirand M, Dellacherie J. 2001. Acides gras et triglycérides du beurre de karité du Cameroun. La Rivista Italiana Delle Sostanze Grasse, LXXVIII: 31-34.

Kapseu C, Womeni HM, Ndjouenkeu R, Tchouanguep MF, Parmentier M. Influence des méthodes de traitement des amandes sur la qualité du beurre de karité. Proc. Biol. Alim., 3: 1-18.

Kapseu C. 2009. Production, analyse et applications des huiles végétales en Afrique. Oléagineux Corps gras et Lipides, 16(4): 215-229.

Karleskind A. 1992. Manuel des Corps Gras (Vol 2). Ed. Technique et Documentation - Lavoisier : Paris; 1579 p. URL : https://books.google.tg/books? $\mathrm{id}=8 \mathrm{KC} 4 \mathrm{ngEACAAJ}$

Karo GB, Baïdi D, Dramane S. 1998. La presse à karité, Projet karité GTZ/DMA, Bamako; 125p.

Kassamba B. 1997. Synthèse des techniques connues d'extraction et de conditionnement du beurre de karité au Burkina Faso. Rapport final, Projet filière karité du CECI (Centre canadien d'Étude et de Coopération Internationale), IRSAT (Institut de Recherche en Sciences Appliquées et Technologie), Ouagadougou, p.3-9.
Konan AG, Nindjin C, Agbo NG, Otokoré DN. 2003. Paramètres de qualité des produits de l'igname en milieu urbain de la Côte d'Ivoire. Agron. Afr., $\mathbf{N}^{\circ}$ spécial (Octobre 2003) : 15-28.

Kouyate AM, Dembele U, Lykke AM. 2015. Les espèces ligneuses locales à huile : une ressource utile pour les communautés locales au Sud du Mali. Int. J. Biol. Chem. Sci., 9(6): 2754-2763. DOI : http://dx.doi.org/10.4314/ijbcs. v9i6.19

Lanoiselle JL, Bouvier M. 1994. Le pressage hydraulique des oléagineux, mise au point. Rev. Franc. des Corps Gras, 3: 61-72.

Lovett P. 2004. La Chaîne de Valeur beurre du karité, Rapport technique WATH $n^{\circ}$ 1, Publication produite pour examen par l'Agence américaine pour le développement international (USAID), $36 \mathrm{p}$.

Lovett PNC. 2004. The shea butter value chain: production, transformation and marketing in West Africa, Technical Report, $\mathrm{N}^{\circ} 2,52 \mathrm{p}$.

Maranz S, Wiesman Z. 2003. Evidence for Indigenous Selection and Distribution of the Shea Tree, Vitellaria paradoxa, and its potential significance to prevailing parkland savanna tree patterns in subSaharan Africa north of the equator. $J$. Biogeogr., 30: 1505-1516.

Maranz S, Wiessman Z, Bisgaard J, Biangui G. 2004. Germoplasm resources of Vitellaria paradoxa based on variations in fats composition across the species distribution range. Agrof. Syst., 60: 7176.

Masters E. 2002. La ressource en karité : vue d'ensemble de la recherche et du développement en Afrique, Atelier $\mathrm{FAO} / \mathrm{CFC}$ sur le traitement, la valorisation et le commerce du karité en Afrique, Sénégal : Centre de suivi 
écologique de Dakar (du 4 au 6 mars 2002) ; 13-31.

Masters ET, Yidana JA, Lovett PN. 2004. Reinforcing Sound Management through Trade: Shea Tree Products in Africa. Unasylva., 210: 46-52.

Mbétid-Bessane E. 2005. Caractérisation du marché des huiles de karité en Centrafrique, Tropicultura, 23(3): 141145.

Nkouam GB. 2007. Conservation des fruits du karité (Vitellaria paradoxa (Gaertn)) et de l'aiéle (Canarium schweinfurthii (Engl.)) : isothermes de sorption d'eau et extraction des matières grasses des fruits stockés, Thèse de Doctorat PhD. Cameroun : Université de Ngaoundéré (ENSAI), 286p.

Soro D, Traore K, Kassi NJ. 2011. Variabilité des caractères morphologiques chez le karité (Vitellaria paradoxa), dans le Nord de la Côte d'Ivoire. Int. J. Biol. Chem. Sci., 5(3): 1201-1214.
Tchabi VI, Adechi KO. 2014. Occupation des terres, typologie et structure des faciès de végétation à Vitellaria paradoxa (Gaertn F.) de la Commune de Toukountouna au Nord - Bénin. Int. J. Biol. Chem. Sci., 8(4): 1684-1696. DOI : http://dx.doi.org/10.4314/ijbcs.v8i4.29

Wolf JP. 1992. Manuel d'Analyse des Corps Gras. Paris : Ed. Tec. et Doc. ; 552.

Womeni HM, Ndjouenkeu R, Kapseu C, Fanni JJ, Parmentier M. 2006. Application du procédé séchage-friture aux amandes de karité : influence sur les indices chimiques de qualité et les propriétés. Oléag. Corp. Gr. Lip., 13: 297-302.

Yé S, Lebeau F, Wathelet J-P, Leemans V, Destain M-F. 2007. Étude des paramètres opératoires de pressage mécanique des amandes de Vitellaria paradoxa Gaertn C. F. (karité), Biotechnol. Agron. Soc. Environ., 11(4): 267-273. 